\section{Atypical Subacute Sclerosing Panencephalitis with Short Onset Latency}

I read with great interest the case reported by the authors [1] describing the unusual latency period in subacute scerosing panencephalitis (SSPE). The usual latency period of SSPE is 6-10 years from its onset as it is a slow virus neurodegenerative disorder. The exact factors and influences that allow the measles infection to persist are unclear, but may include several immunological factors [2]. Numerous alterations in $M$ protein have been described in SSPE because of extensive point mutations in viral genome, possibly resulting in persistent viral infection [3].

Since it is a slow progressive neurodegerative disorder a latency period of two month is very unusual. Authors have not described the pathogenesis behind this unusual occurrence. Moreover CSF analysis shows only raised IgG levels with normal IgM levels but looking at short latency period there is more possibility of raised IgM levels instead of IgG measles antibody. Therefore this case is looking more likely a case of SSPE from congenital measles and in that case this latency becomes irrelevant. Serology of the mother can be helpful in these cases.

DEEPAK SACHAN
Associate Professor,
Department of Pediatrics, PGIMER and Associate
DR RML hospital, NewDelhi110001, India.
deepaksachan26@yahoo.com

\section{REFERENCES}

1. Saurabh K, Gupta R, Khare S, Sharma S. Atypical subacute sclerosing panencephalitis with short onset latency. Indian Pediatr. 2013;50:244-5.

2. Garg RK. Subacute sclerosing panencephalitis. J Neurol. 2008;255:1861-71.

3. Hayashi M, Arai N, Satoh J, Suzuki H, Katayama K, Tamagawa K, et al. Neurodegenerative mechanisms in subacute sclerosing panencephalitis. J Child Neurol. 2002;17:725-30.

\section{Cholera-like Illness Due to Aeromonas caviae}

A 2-year-old girl presented with rice watery stools and vomiting for 12 hours. There was no blood in the stools. On examination she had some dehydration. Stool microscopy and hanging drop revealed darting motile bacilli morphologically resembling Vibrio cholera. Her blood count and renal function tests were normal. Dehydration was corrected with IV Ringer lactate and she was commenced on Azithromicin. Her HIV status was non-reactive. Stool culture grew Aeromonas caviae, which was sensitive to doxycycline, chloramphenicol, furazolidine and resistant to nalidixic acid, ceftraixone, co-trimaxazole and ampicillin. She was discharged after three days.

Aeromonas species are ubiquitous water borne medically important, Gram-negative, rod-shaped microorganisms [1]. Today, they are regarded not only as disease-causing pathogen of fish but are also responsible for variety of complications in both immunocompetent and immunocompromised humans [2]. Aeromonas have gained importance as human pathogens causing gastrointestinal infections. They also cause extraintestinal infections such as cellulitis, wound infections, sepsis and urinary tract infections [1]. Deodhar, et al. [3] isolated Aeromonas from 45 (1.8\%) of 2,480 patients with acute gastroenteritis. Out of 863 traveller's diarrhea patients returning from Asia, Africa, and Latin America, 2\% of cases were caused by Aeromonas [4]. Studies have shown that three Aeromonas (A. hydrophila, A. caviae and A. veronii bv Sobria) are responsible for $\geq 85 \%$ of human infections [2]. Clinical spectrum of Aeromonas-induced diarrhoea varies from toxigenic diarrhea to colitis and in developing countries it is predominantly toxigenic [3]. The most common presentation for Aeromonas gastroenteritis is secretory(watery) enteritis suggesting the toxigenic nature of the organism [2]. Aeromonas strains are almost universally susceptible to fluoroquinolones and exhibited multidrug resistance [2]. Bhowmika, et al. [1] isolated potentially pathogenic and 